\title{
Molecular and epidemiological characterization of human adenovirus and classic human astrovirus in children with acute diarrhea in Shanghai, 2017-2018
}

\author{
Lijuan Lu, Huaqing Zhong, Menghua Xu, Liyun Su, Lingfeng Cao, Ran Jia and Jin Xu*
}

\begin{abstract}
Background: In addition to rotavirus and norovirus, human adenovirus (HAdV) and classic human astrovirus (classic HAstV) are important pathogens of acute diarrhea in infants and young children. Here, we present the molecular epidemiology of HAdV and classic HAstV in children with acute diarrhea in Shanghai.

Methods: Fecal specimens were collected from 804 outpatient infants and young children diagnosed with acute diarrhea in Shanghai from January 2017 to December 2018. All of the samples were screened for the presence of HAdV and classic HAstV. HAdV and classic HAstV were detected using traditional PCR and reverse-transcription PCR, respectively. All of the HAdV and classic HAstV positive samples were genotyped by phylogenetic analysis.

Results: Among the 804 fecal samples, 8.58\% (69/804) of samples were infected with either HAdV or classic HAstV, and five were co-infected with two diarrhea viruses. The overall detection rates of HAdV and classic HAstV were $3.47 \%(28 / 804)$ and $5.22 \%(42 / 804)$, respectively. Four subgroups (A, B, C, and F) and seven genotypes (HAdV-C1, $-C 2,-B 3,-C 5,-A 31,-F 40$, and $-F 41)$ of HAdV were detected. Subgroup $F$ had the highest constituent ratio at 64.29\% (18/28), followed by non-enteric HAdV of subgroup C $(21.43 \%, 6 / 28)$ and subgroup B $10.71 \%(3 / 28)$. HAdVF41 $(60.71 \%, 17 / 28)$ was the dominant genotype, followed by HAdV-C2 $(14.29 \%, 4 / 28)$ and HAdV-B3 $(10.71 \%, 3 / 28)$. Two genotypes of classic HAstV (HAstV-1 and HAstV-5) were identified in 42 samples during the study period; HAstV-1 (95.24\%, 40/42) was the predominant genotype, and the other two strains were genotyped as HAstV-5. No significant differences were found between boys and girls in the detection rates of HAdV $(P=0.604)$ and classic HAstV $(P=0.275)$. Over half of the HAdV infections $(82.14 \%, 23 / 28)$ and classic HAstV infections $(66.67 \%, 28 / 42)$ occurred in children less than 36 months. Seasonal preferences of HAdV and classic HAstV infections were summer and winter, respectively. In this study, the common clinical symptoms of children with acute diarrhea were diarrhea, vomiting, fever and abdominal pain.
\end{abstract}

Conclusions: Our findings indicate that HAdV and classic HAstV play important roles in the pathogenesis of acute diarrhea in children in Shanghai. Systematic and long-term surveillance of HAdV and classic HAstV are needed to monitor their prevalence in children and prevent major outbreak.

Keywords: Human adenovirus, Human astrovirus, Children, Diarrhea, Genotype

\footnotetext{
* Correspondence: jinxu_125@163.com

Department of Clinical Laboratory, Children's Hospital of Fudan University,

399 Wanyuan Road, Shanghai 201102, China
}

(c) The Author(s). 2021 Open Access This article is licensed under a Creative Commons Attribution 4.0 International License, which permits use, sharing, adaptation, distribution and reproduction in any medium or format, as long as you give appropriate credit to the original author(s) and the source, provide a link to the Creative Commons licence, and indicate if changes were made. The images or other third party material in this article are included in the article's Creative Commons licence, unless indicated otherwise in a credit line to the material. If material is not included in the article's Creative Commons licence and your intended use is not permitted by statutory regulation or exceeds the permitted use, you will need to obtain permission directly from the copyright holder. To view a copy of this licence, visit http://creativecommons.org/licenses/by/4.0/ The Creative Commons Public Domain Dedication waiver (http://creativecommons.org/publicdomain/zero/1.0/) applies to the data made available in this article, unless otherwise stated in a credit line to the data. 


\section{Background}

Acute diarrhea is one of the major health problems in children under 5 years old. Approximately 1.0 billion children $<5$ years of age are infected with diarrheal diseases, and 0.45 million deaths occur due to diarrhea each year [1-3]. Diarrhea can be caused by various types of viruses, bacteria, and parasites. Viruses have long been considered the most important pathogens responsible for acute gastroenteritis, with rotavirus group A and norovirus being the most prominent causes of acute diarrhea in children [4-6]. Human adenovirus and classic human astrovirus are also recognized as important causes of sporadic diarrhea and outbreaks of diarrhea in children $[7,8]$.

Human adenovirus (HAdV) belongs to the genus Mastadenovirus of the family Adenoviridae. HAdV is non-enveloped, double-stranded, 26-45 kbp linear DNA viruses that possess an outer capsid and inner core structural proteins. The outer capsid comprises fiber proteins, penton, and hexon. The fiber proteins are attached to the penton base, and penton is the secondmost abundant component consisting of 12 penton bases. The hexon is the principal component of the capsid. HAdV are categorized into seven species (HAdV-A through HAdV-G) based on genomic sequence analysis, and more than 100 genotypes have been recognized [911]. Different genotypes have been identified by multiplex PCR techniques and sequencing of targeting fiber genes or hexon genes $[12,13]$. HAdV infections lead to disease of several human systems, including acute respiratory illness, acute gastroenteritis, conjunctiva, hemorrhagic cystitis, hepatitis, hemorrhagic colitis, pancreatitis, nephritis, and meningoencephalitis [13]. Genotypes 40 and 41 of HAdV-F are the most frequently reported causes of HAdV-associated diarrhea in young children and are known as enteric HAdV. Indeed, HAdV-40 and 41 are responsible for $1-20 \%$ of diarrhea cases in both outpatients and hospitalized children worldwide [14-18]. Some cases of acute diarrhea in children have been reported to be associated with HAdV$12,-18$, and -31 of HAdV-A. Moreover, HAdV-B, HAdV-C, HAdV-D, and HAdV-G have also been detected in fecal samples from children with acute gastroenteritis $[14,15,18,19]$.

Human astrovirus (HAstV) belongs to the Astroviridae family, which is divided into two genera, Mamastrovirus and Avastrovirus, based on their ability to infect mammalian and avian species, respectively. HAstV is nonenveloped, positive sense, single-stranded RNA viruses. The HAstV genome is $6.8-7.9 \mathrm{~kb}$ in length and consists of a $5^{\prime}$ untranslated region (UTR), followed by three open reading frames (ORFs) (ORF1a, ORF1b, and ORF2), a 3' UTR, and a poly A tail. ORF1a and ORF1b encode nonstructural proteins, including the RNA- dependent RNA polymerase (RdRp), while ORF2 encodes the capsid protein precursor [20]. The initial prototype strain of the human astrovirus species was originally isolated in 1975, and is known as the classic human astrovirus (classic HAstV). With the development of next-generation sequencing technologies, two novel groups of highly divergent HAstV, Melbourne (MLB) and Virginia/Human-Mink-Ovine-like (VA/HMO), have been identified in human stools of individuals with diarrhea worldwide $[7,21,22]$. The overall detection rate of novel HAstV in stools was much lower than that of classic HAstV, which remains the second or third most common viral pathogen responsible for diarrhea in young children. Until now, eight genotypes of classic HAstV (HAstV-1 to HAstV-8) have been identified [23]. Globally, classic HAstV is responsible for $2-18.8 \%$ of cases of acute diarrhea in children. HAstV-1 is the most prevalent genotype detected in children, whereas HAstV-2-HAstV-8 are less prevalent [14, 20, 24-26].

In Shanghai, the majority of previous studies have focused on the molecular and epidemiological characteristics of rotavirus and norovirus, while relatively few studies have been conducted on the molecular epidemiology of HAdV and classic HAstV in outpatient [14, 2729]. Therefore, we sought to investigate the detection rate, viral co-infection, seasonal distribution, age distribution, and genetic diversity of HAdV and classic HAstV infections in children with acute diarrhea in Shanghai from 2017 to 2018 .

\section{Materials and methods Study design}

From 2017 to 2018, a total of 804 stool specimens were collected from children $<5$ years who were diagnosed with acute diarrhea and admitted to the outpatient department of the Children's Hospital of Fudan University, Shanghai, China. All of the enrolled specimens were routinely collected and stored at $-70{ }^{\circ} \mathrm{C}$ prior to investigation. The definition of acute diarrhea was three or more loose, watery, thin stools with a paste-like texture, or the presence of mucous stools within $24 \mathrm{~h}$, possibly accompanied by vomiting, abdominal pain, fever, and nausea. This definition excluded the presence of pus or blood regardless of the presence of fever [14]. Demographic information and clinical diagnoses were gathered from the children's medical histories. Informed consent was not required from the patients because the stool specimens were collected during the normal course of patient care. The study proposal was approved by the Institutional Review Board of the Children's Hospital of Fudan University. All methods were carried out in accordance with the relevant guidelines and regulations.

Viral genomic RNA and DNA were extracted from $10 \%$ fecal suspension supernatant using the TIANamp 
Virus DNA/RNA Kit (Tiangen Biotech, Beijing, China) according to the manufacturer's instructions. Extracted genetic material was reverse transcribed into cDNA with a random primer using Prime-Script ${ }^{\mathrm{Tm}}$ II Reverse Transcriptase (Takara, Biotechnology [Dalian] Co., Ltd.) for detection of the Classic HAstV. A conserved region (C4) in the HAdV hexon gene was amplified using the Ad-1 (5' ${ }^{\prime}$ TTCCCC-ATGGCICAYAACAC-3') and Ad-2 (5'CCCTGGTAKC-CRATRTTGTA-3') primers [30]. The expected size of the amplicon was $482 \mathrm{bp}$. The PCR cycling program was as follows: an initial denaturation at $94{ }^{\circ} \mathrm{C}$ for $2 \mathrm{~min}$, followed by 35 cycles of $30 \mathrm{~s}$ at $94{ }^{\circ} \mathrm{C}, 30$ s at $55^{\circ} \mathrm{C}$, and $1 \mathrm{~min}$ at $72{ }^{\circ} \mathrm{C}$, with a final extension cycle at $72^{\circ} \mathrm{C}$ for $7 \mathrm{~min}$. Classic HAstV in fecal specimens was detected using primers Mon269 (5'-CAAC TCAGGAAACAGGGTGT-3') and Mon270 (5'-CTGG CTTAACCCACATTCC-3'), which targeted the ORF2 region $C$ [31]. The expected size of the PCR product was $449 \mathrm{bp}$. PCR amplification was performed under the following conditions: $94{ }^{\circ} \mathrm{C}$ for $2 \mathrm{~min}, 35$ cycles of $94^{\circ} \mathrm{C}$ for $30 \mathrm{~s}, 55^{\circ} \mathrm{C}$ for $30 \mathrm{~s}$, and $72{ }^{\circ} \mathrm{C}$ for $1 \mathrm{~min}$ followed by $72{ }^{\circ} \mathrm{C}$ for $7 \mathrm{~min}$. All of the PCR products were electrophoresed in a $2 \%$ agarose gel with ethidium bromide and a DNA ladder of $100 \mathrm{bp}$ (Takara Bio Co., Dalian, China).

All the amplicons of HAdV and classic HAstV were purified and sequenced for phylogenetic analysis by firstgeneration sequencing technologies (Sangon Biotech [Shanghai] Co., Ltd.). Phylogenetic trees were constructed using the maximum likelihood method (Kimura two parameters substitution model with 1000 bootstrap replications for branch support) in MEGA (v6.0) software. The nucleotide sequences of HAdV and classic HAstV detected in this study were compared to the sequences of corresponding reference virus strains available in the GenBank database.

The nucleotide sequences of HAdV strains and the accession numbers used were as follows: HAdV-1: AC_ 000017, AF534906; HAdV-2: J01917, AC_000007; HAdV-3: AY599836; HAdV-4: AY487949; HAdV-5: AY339865; HAdV-8: AB448768; HAdV-9: AJ854486; HAdV-11: AY163756; HAdV-12: X73487; HAdV-14: AY803294; HAdV-16: AY601636; HAdV-17: AF108105; HAdV-21: AY601633; HAdV-22: FJ404771; HAdV-26: EF153474; HAdV-28: FJ824826; HAdV-29: AB562587; HAdV-31: AM749299; HAdV-34: AY737797; HAdV-35: AY128640; HAdV-36: GQ384080; HAdV-37: AB448777; HAdV-40: L19443; HAdV-41: DQ315364; HAdV-46: AY875648; HAdV-48: EF153473; HAdV-49: DQ393829; HAdV-53: AB605240; HAdV-54: NC 012959; HAdV-A: NC_001460; HAdV-B: NC_011203; HAdV-C: NC_ 001405; HAdV-D: AC_010956; HAdV-E: NC_003266; and HAdV-F: NC_001454. The reference classic HAstV strains and accession numbers used were as follows: HAstV-1: L23513, Z25771; HAstV-2: L13745; HAstV-3:
AFl41381, L38505; HAstV-4: AY720891， L38506; HAstV-5: DQ028633, U15136; HAStV-6: L38507, Z46658; HAstV-7: L38508, Y08632; HAstV-8: AF260508, Z66541.

\section{Statistical analysis}

Statistically significant differences in infection rates of categorical variables were tested using Fisher's exact test, two-sided chi-square test and corrected chi-square test in SPSS Statistics v.20.0 (IBM Corp., Armonk, NY, USA). $P$-values $<0.05$ were considered as statistically significant.

\section{Results}

\section{Prevalence of HAdV and classic HAstV infections}

During the study period, a total of 804 stool samples from children with acute diarrhea were enrolled in our study. Among them, 497 were boys and 307 were girls, and all of the children had been diagnosed with acute diarrhea at the Children's Hospital of Fudan University in Shanghai following attendance as outpatients.

Among these 804 fecal samples, 8.58\% (69/804) were infected with HAdV or classic HAstV, and five patients were co-infected with two viruses (Table S1 and Table S2). The overall detection rates of HAdV and classic HAstV were $3.47 \%(28 / 804)$ and 5.22\% (42/804), respectively. The frequency of HAdV in boys and girls was $3.22 \%(16 / 497)$ and $3.91 \%(12 / 307)$, respectively $(P=$ 0.604). The prevalence of classic HAstV in boys and girls was $5.84 \%(29 / 497)$ and $4.23 \%(13 / 307)$, respectively $(P=0.275)$. The prevalence of HAdV in 2017 and 2018 was $2.84 \%(12 / 423)$ and $4.20 \%(16 / 381)$, respectively $(P=0.293)$. The annual detection rates of classic HAstV varied significantly according to the year as follows: $2.84 \%(12 / 423)$ in $2017,7.87 \%(30 / 381)$ in $2018(P=$ $0.001)$.

\section{Seasonal and age distribution of HAdV- and classic HAstV-infected children}

The seasonal distribution of HAdV peaked in the June of both 2017 (18.75\%, 6/32) and 2018 (15.38\%, 4/26). During the study period, HAdV was detected in 13 of the total 24 months (Fig. 1). The peak of classic HAstV was December 2017 (11.76\%, 4/34) and November 2018 (33.33\%, 10/30). Classic HAstV was not detected in 10 of the total 24 months (Fig. 1).

Infections of HAdV and classic HAstV were found in all age groups. Approximately 82.14\% (23/28) of HAdVinfected cases and over half of classic HAstV-infected children $(66.67 \%, 28 / 42)$ were found in children $<36$ months. The group of children between 37 and 48 months old had the highest prevalence of HAdV infections $(13.33 \%, 6 / 45)$ and HAstV infections $(5.17 \%, 3 / 55)$ (Fig. 2). 


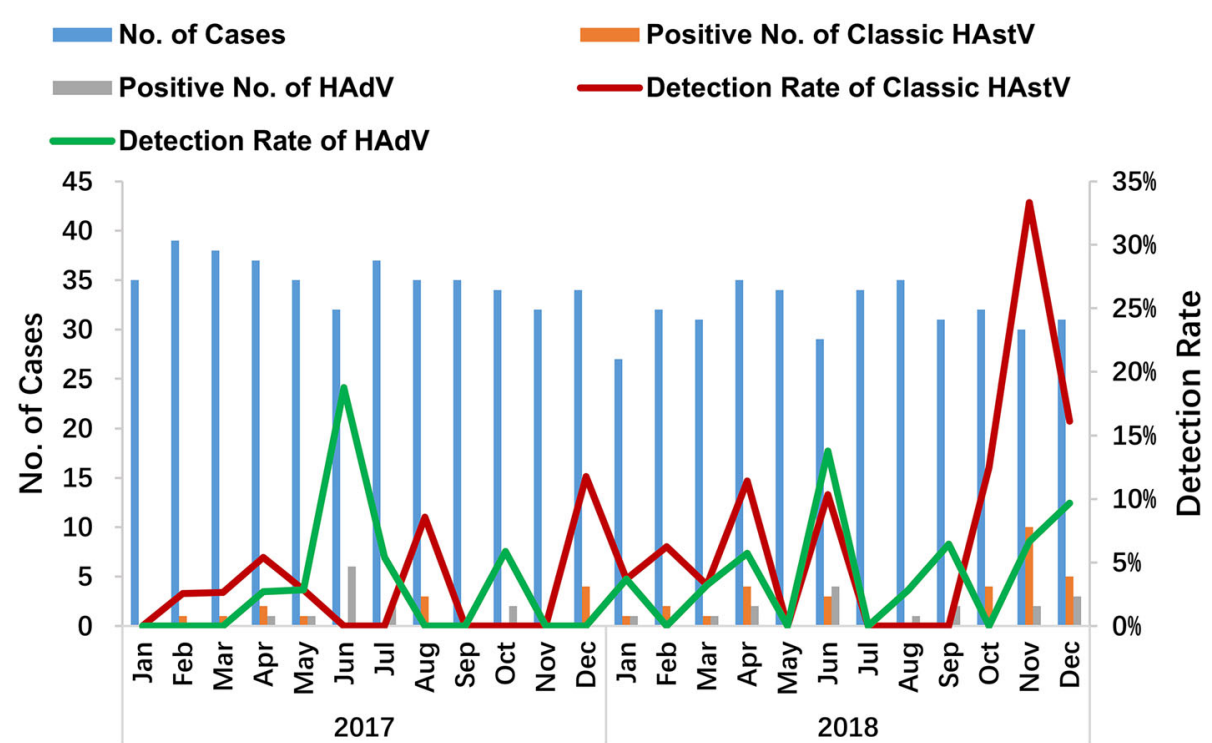

Fig. 1 Seasonal distribution of HAdV and classic HAstV genotypes detected in the current study

\section{Genotypes of HAdV and classic HAstV infections}

During the course of the study period, a total of 28 and 42 nucleotide sequences of HAdV and classic HAstV were obtained, respectively. The phylogenetic trees of nucleotide sequences of the HAdV and classic HAstV isolates were constructed in comparison to the reference strains.

According to the phylogenetic tree analysis conducted based on a partial genomic region of hexon, four subgroups of HAdV (A, B, C, and F) were detected, and seven different genotypes (HAdV-A31, -B3, -C1, -C2, $-\mathrm{C} 5,-\mathrm{F} 40$ and -F41) were identified. Subgroup F, classified as enteric HAdV, had the highest constituent ratio at $64.29 \%(18 / 28)$, followed by non-enteric HAdV of subgroup C $(21.43 \%, 6 / 28)$ and subgroup B $(10.71 \%, 3 /$ $28)$. Of the seven genotypes, HAdV-F41 (60.71\%, 17/28) was the dominant genotype, followed by HAdV-C2 $(14.29 \%, 4 / 28)$ and HAdV-B3 $(10.71 \%, 3 / 28)$. HAdV-F41

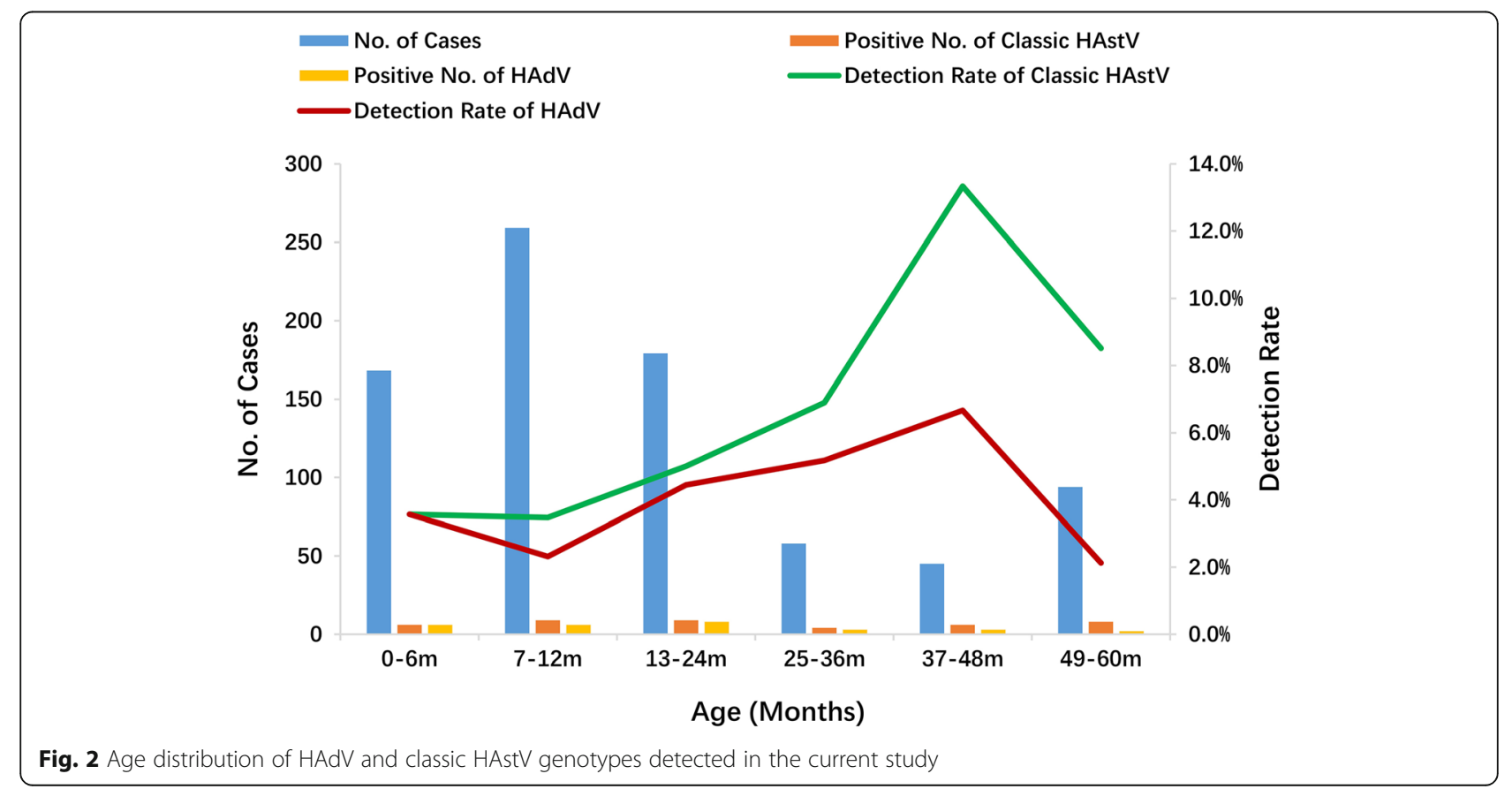


was the most common genotype at $83.33 \%(10 / 12)$ and $43.75 \%(7 / 16)$ in 2017 and 2018, respectively. The second most prevalent genotype varied from 2017 to 2018 . HAdV-C2 $(16.67 \%, 2 / 12)$ was the second most prevalent genotype in 2017, while HAdV-B3 $(18.75 \%, 3 / 16)$ was the most prevalent in 2018. Of note, only two genotypes (HAdV-C2 and HAdV-F41) were identified in 2017, while all the seven HAdV genotypes were detected in 2018 (Fig. 3).

Based on the ORF2 region C of classic HAstV, two different genotypes of classic HAstV (HAstV-1 and HAstV-5) were identified in 42 samples during the study period. HAstV-1 $(95.24 \%, 40 / 42)$ was the predominant genotype in this study and was the only genotype detected in 2017. In addition to HAstV-1 (93.33\%, 28/30), HAstV-5 (6.67\%, 2/30) was also identified in 2018 (Fig. 4).

\section{Clinical features of children infected with HAdV and classic HAstV}

The most common clinical symptom of HAdV infected patients was diarrhea $(100.0 \%, 28 / 28)$, followed by vomiting $(35.7 \% \%, 10 / 28)$, fever $(25.0 \%, 7 / 28)$ and abdominal pain $(3.6 \%, 1 / 28)$. The common clinical symptoms of classic HAstV infected patients were diarrhea $(100.0 \%, 42 / 42)$, fever $(26.2 \%, 11 / 42)$, vomiting $(23.8 \%$, $10 / 42)$ and abdominal pain $(7.1 \%, 3 / 42)$ (Table 1$)$. The difference between infected and uninfected classic HAstV group $(P=0.032)$ was statistically significant when the clinical symptoms of diarrhea and abdominal pain diarrhea occurred at the same time in children (Table 1). No significant differences were observed in clinical features among children with acute diarrhea under other infection circumstances (Tables 1 and 2, Tables S1 and Table S2).

\section{Discussion}

Although HAdV and classic HAstV generally cause a self-limiting short-term watery diarrhea, they are frequent causes of acute diarrhea in children $<5$ years of age $[3,20]$. Real-time monitoring of HAdV and classic HAstV can assist with monitoring their prevalence in children with acute gastroenteritis and, as a result, could play a guiding role in the prevention of major epidemics in Shanghai.

The overall stool positivity rate for HAdV infection in the present study was $3.47 \%$, which is similar to that previously reported in Brazil (3.9\%), Bangladesh (4.82\%), and in our previous study (5.2\%), but is much lower than that reported in Northwest Ethiopia (32.0\%) and Albania (23.2\%) [18, 24, 32-34]. According to our continuous monitoring data, the detection rate of HAdV in children with acute diarrheas was relatively stable in Shanghai from 2010 to 2018 [14]. In addition to the data from our previous study from 2010 to 2011 (1.9\%), the detection rate of classic HAstV (5.22\%) in Shanghai was also lower than the average global positive rate of $11.0 \%$ [14, 20]. This frequency is similar to that observed in other studies from Thailand (2.6\%), Asian Russia (2.8\%), Brazil (3.9\%), Lebanon (5.5\%), and Germany (5.0\%) [35-39]. However, the detectable rate of classic HAstV in 2018 (7.87\%) was significantly higher than the detection rate in $2017(2.84 \%)$ in the current study, and long-term monitoring is needed to determine the reason for this increase. Furthermore, sex was not found to play a role in HAdV and classic HAstV infections in our study, which is consistent with the findings of studies in Tanzania and Northwest Ethiopia [24, 40].

Although only a small number of positive samples of HAdV and classic HAstV were reported in this study, data on the seasonality of infection with these two viruses were also analyzed. As a result, we found that HAdV and classic HAstV infections had a tendency to occur in oscillatory fluctuations. The highest rates of HAdV infection were observed in July of both 2017 and 2018, which was similar to the rates observed in Tianjin from 2008 to 2009, and Thailand from 2011 to 2017 [8, 41]. However, in our previous study on patients with acute diarrhea from 2006 to 2011, HAdV infection was more frequent during the winter months [27]. Moreover, no seasonal pattern of HAdV infection was observed in our previous study on outpatients from 2012 to 2016 [36]. Taken together, these data indicate that the seasonal pattern of HAdV infection is consistent in Shanghai. A longer time-series analysis is needed to describe the discrepancies in HAdV prevalence drawn from the acquired data of inpatients and outpatients $\leq 5$ years of age. The same lack of seasonal pattern was found in Thailand and India [8, 42]. Similar to several other studies conducted in Germany, Spain, Northern Italy, and our previous study, classic HAstV infection was common during the cold-weather period in Shanghai [14, 26, 39, 43].

According to our data, higher HAdV $(82.14 \%, 23 / 28)$ and classic HAstV $(66.67 \%, 28 / 42)$ positive component ratios were identified in children $\leq 3$ years, which is in line with the findings of other studies [19, 27, 32]. In this study, HAdV $(13.33 \%, 6 / 45)$ and classic HAstV (6.67\%, $3 / 42$ ) infection were most commonly detected in children $37-48$ months old. This finding suggests that herd immunity to HAdV and classic HAstV may develop gradually in children $>4$ years old in Shanghai. However, the neutralizing antibody production, duration of herd immunity, and the epidemiological pattern to HAdV and classical HAstV remain to be determined.

Molecular characterization of HAdV through phylogenetic analysis revealed genetic diversity in the samples analyzed in this study. A total of seven HAdV genotypes, 


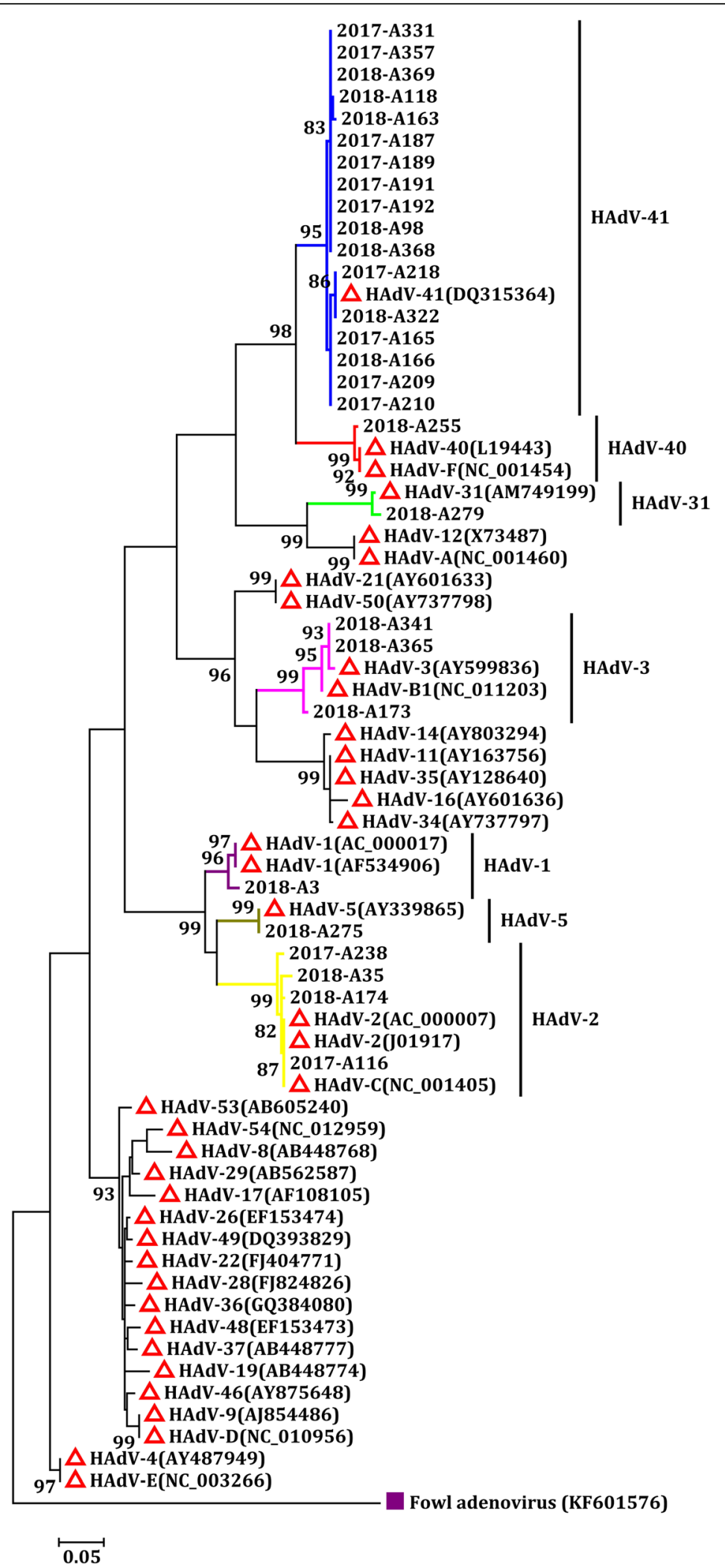

Fig. 3 Phylogenetic analysis of partial hexon gene sequences of HAdV detected in children. $\triangle$ Reference strains 


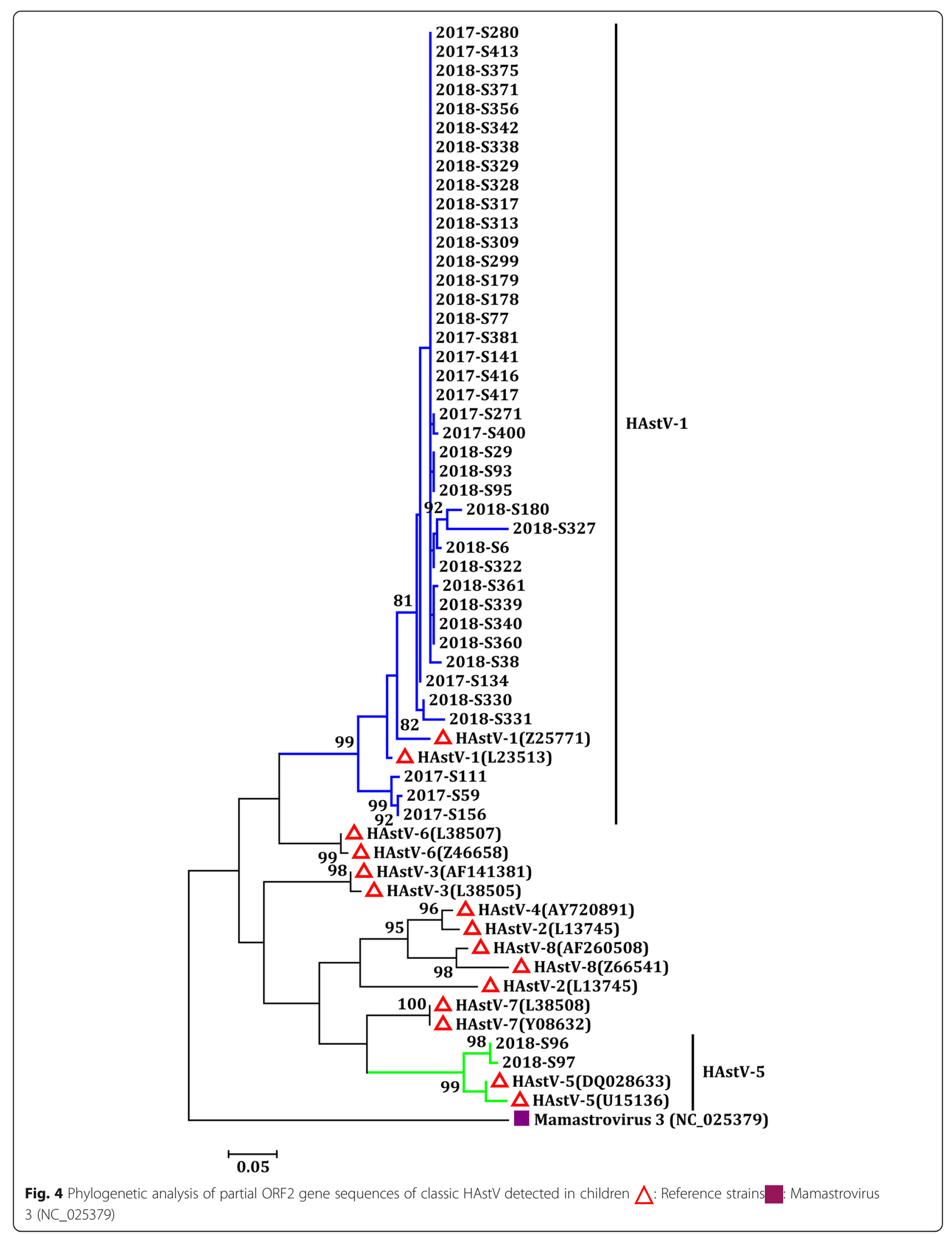


Table 1 Clinical symptoms of diarrhea children infected with and without HAdV or classic HAstV

\begin{tabular}{|c|c|c|c|c|}
\hline \multirow[t]{2}{*}{ Clinical symptoms } & \multicolumn{2}{|c|}{ HAdV, n (\%) } & \multicolumn{2}{|c|}{ classic HAstV, n(\%) } \\
\hline & Positive & Negative & Positive & Negative \\
\hline Diarrhea only & $13(46.4)$ & $486(62.6)$ & $20(47.6)$ & $474(62.2)$ \\
\hline Diarrhea and vomiting & $7(25.0)$ & $114(14.7)$ & $8(19.1)$ & $120(15.8)$ \\
\hline Diarrhea and fever & $4(14.3)$ & $100(12.9)$ & $9(21.4)$ & $104(13.6)$ \\
\hline Diarrhea and abdominal pain & $1(3.6)$ & $16(2.0)$ & $3(7.1)$ & $11(1.4)^{a}$ \\
\hline Diarrhea, vomiting and fever & $3(10.7)$ & $54(7.0)$ & $2(4.8)$ & $48(6.3)$ \\
\hline Diarrhea, vomiting and abdominal pain & $0(0)$ & $2(0.3)$ & 0 & $1(0.1)$ \\
\hline Diarrhea, fever and abdominal pain & $0(0)$ & $1(0.1)$ & 0 & $2(0.3)$ \\
\hline Diarrhea, vomiting, fever and abdominal pain & $0(0)$ & $3(0.4)$ & 0 & $2(0.3)$ \\
\hline Total & $28(100.0)$ & 776 (100.0) & $42(100.0)$ & $762(100.0)$ \\
\hline
\end{tabular}

${ }^{a}$ The difference between infected and uninfected classic HAstV group was statistically significant when the clinical symptoms of diarrhea and abdominal pain occurred at the same time $(P=0.032)$

including five non-enteric HAdV genotypes, were found in children with acute diarrhea from 2017 to 2018. Our survey of HAdV genotypes in children with acute diarrhea indicated that enteric HAdV, including HAdV-F40 and HAdV-F41, accounted for $64.29 \%$ (18/28), and can therefore be considered the most prevalent pathogens associated with acute diarrhea in Shanghai. However, HAdV-F40 was only found in one child; this finding coincides with those from our previous studies, and those in Bangladesh and Japan [14, 32, 44]. One reason for the predominance of HAdV-F41 over HAdV-F40 is antigenic drift of HAdV-F41. Meanwhile, some studies have discovered that GTC1 and GTC2 subdivisions trigged by the build-up of amino acid mutations in the HVRs (hexon hypervariable regions) of hexon may allow HAdV-F41 to escape from the host immune response, leading to increased HAdV-F41 infection [45-47].

The results of this study suggest that non-enteric HAdV, including HAdV-A31, $-\mathrm{B} 3,-\mathrm{C} 1,-\mathrm{C} 2$, and $-\mathrm{C} 5$, play important roles in causing acute diarrhea in children, although they primarily caused conjunctiva and upper and lower respiratory tract infections [13]. Interestingly, non-enteric HAdV-C2 and HAdV-B3 infections unexpectedly exceeded that of HAdV-F40 and became the second and third leading genotype in children with acute diarrhea, respectively. In addition, in contrast to our previous studies from 2012 to 2016, the detection rate of HAdV-C2 exceeded that of HAdV-A31 and was therefore found to be the second most prevalent genotype from 2017 to 2018 [39]. Taken together, these results suggest that the genotypes of non-enteric HAdV in children with acute diarrhea undergo dynamic changes in Shanghai, demonstrating the importance of continuous surveillance of HAdV in this patient group.

HAstV-1 is the most prevalent classic HAstV genotype detected worldwide, whereas HAstV-2-HAstV-8 are less prevalent $[7,23]$. According to the phylogenetic tree analysis of classic HAstV, only two genotypes, including HAstV-1 and HAstV-5, were identified in Shanghai from 2017 to 2018. HAstV-1 (95.24\%) was the predominant genotype detected in children with diarrhea, which is consistent with the findings of our previous study from 2008 to 2011 as well as with other reports conducted in Japan, Switzerland, Asian Russia, Korea, Germany, and Brazil [36, 37, 39, 48-51]. Moreover, HAstV-5 was only detected in two samples in early 2018; to the best of our knowledge, this study is the first to report the appearance of HAstV-5 in Shanghai. Nevertheless, long-term monitoring data on HAstV-5 are needed to derive the epidemic characteristics of this genotype.

Typical clinical symptoms of these children with acute diarrhea were diarrhea, vomiting, fever, and abdominal

Table 2 Clinical features observed among diarrheic children infected with HAdV and classic HAstV

\begin{tabular}{llll}
\hline Clinical symptoms & $\begin{array}{l}\text { Enteric HAdV, } \\
\mathbf{n}(\%)\end{array}$ & $\begin{array}{l}\text { Non-enteric HAdV, } \\
\mathbf{n}(\%)\end{array}$ & $\begin{array}{l}\text { Classic HAstV, } \\
\mathbf{n}(\%)\end{array}$ \\
\hline Diarrhea only & $10(55.5)$ & $3(30.0)$ & $20(47.6)$ \\
Diarrhea and vomiting & $4(22.2)$ & $3(30.0)$ & $8(19.1)$ \\
Diarrhea and fever & $2(11.1)$ & $2(20.0)$ & $9(21.4)$ \\
Diarrhea and abdominal pain & $1(5.6)$ & $0(0)$ & $3(7.1)$ \\
Diarrhea, vomiting and fever & $1(5.6)$ & $2(20.0)$ & $2(4.8)$ \\
Total & $18(100)$ & $10(100)$ & $42(100)$ \\
\hline
\end{tabular}


pain. This finding was in agreement with the previous reports of viruses infected patients [32, 50]. Moreover, the results of this study suggest that children infected with classic HAstV would be more likely to experience abdominal pain compared with HAstV negative children.

\section{Conclusions}

In the current study, we clarified the epidemiological role of HAdV and classic HAstV in children < 5 years with acute diarrhea in Shanghai from 2017 to 2018. HAdV-41 has a significant involvement in the etiology of acute diarrhea in children $<5$ years; however, the role of non-enteric HAdV in children cannot be ignored. We also found that HAstV-1 was the most predominant genotype in Shanghai. These findings enhance our knowledge of the significance of HAdV and classic HAstV infections in children.

\section{Abbreviations \\ HAdV: Human adenovirus; HAstV: Human astrovirus; ORFs: Open reading frames; UTR: Untranslated region; RdRp: RNA-dependent RNA polymerase; MLB: Melbourne; VA/HMO: Virginia/Human-Mink-Ovine-like; MEGA: Molecular evolutionary genetics analysis; GTC: Genome-type clusters; HVRs: Hexon hypervariable regions}

\section{Acknowledgments}

We thank the participants for their contributions to this study.

\section{Authors' contributions}

$J X$ and $L J L$ conceived and designed the study. $L J L$ performed the experiments and wrote the manuscript. HQZ and MHX assisted in the data analysis and interpretation. LYS, LFC, and RJ acquired the clinical data and coordinated the study design. All authors have read and approved the final manuscript.

\section{Funding}

None.

\section{Availability of data and materials}

The datasets used in the current study are available from the following link: http://purl.org/phylo/treebase/phylows/study/TB2:S27343?x-access-code= $3821 \mathrm{~b} 7362045 \mathrm{cab03c705697 \textrm {db } 4 3 7 f 1 3 \& f o r m a t = h t m l}$ and the accession number is 27343 .

\section{Declarations}

\section{Ethics approval and consent to participate}

This study and informed consent waiver statement were reviewed and approved by the Institutional Review Board of the Children's Hospital of Fudan University. Consent was not sought from the parent or legal guardians of the children because the fecal specimens enrolled in this study were left over after routine examination.

\section{Consent for publication}

Not applicable.

\section{Competing interests}

The authors have no competing interest.

Received: 26 November 2020 Accepted: 18 July 2021

Published online: 29 July 2021

\section{References}

1. Sharif N, Nobel NU, Sakib N, Liza SM, Khan ST, Billah B, et al. Molecular and epidemiologic analysis of diarrheal pathogens in children with acute gastroenteritis in Bangladesh during 2014-2019. Pediatr Infect Dis J. 2020; 39(7):580-5. https://doi.org/10.1097/INF.0000000000002637.

2. Kirk MD, Pires SM, Black RE, Caipo M, Crump JA, Devleesschauwer B, et al. World Health Organization estimates of the global and regional disease burden of 22 foodborne bacterial, protozoal, and viral diseases, 2010: a data synthesis. PLoS Med. 2015;12(12):e1001921. https://doi.org/10.1371/journal. pmed.1001921.

3. Lanata CF, Fischer-Walker CL, Olascoaga AC, Torres CX, Aryee MJ, Black RE. Child health epidemiology reference Group of the World Health O, Unicef: global causes of diarrheal disease mortality in children $<5$ years of age: a systematic review. PLoS One. 2013;8(9):e72788. https://doi.org/10.1371/ journal.pone.0072788,

4. Cohen R, Raymond J, Gendrel D. Antimicrobial treatment of diarrhea/acute gastroenteritis in children. Arch Pediatr. 2017;24(12):S26-9. https://doi.org/1 0.1016/S0929-693X(17)30515-8

5. Yu J, Lai S, Geng Q, Ye C, Zhang Z, Zheng Y, et al. Prevalence of rotavirus and rapid changes in circulating rotavirus strains among children with acute diarrhea in China, 2009-2015. J Inf Secur. 2019;78(1):66-74.

6. Bányai K, Estes MK, Martella V, Parashar UD. Viral gastroenteritis. Lancet. 2018;392(10142):175-86. https://doi.org/10.1016/S0140-6736(18)31128-0.

7. Johnson C, Hargest V, Cortez V, Meliopoulos VA, Schultz-Cherry S. Astrovirus pathogenesis. Viruses. 2017;9(1):22. https://doi.org/10.3390/v9010022.

8. Kumthip K, Khamrin P, Ushijima H, Maneekarn N. Enteric and non-enteric adenoviruses associated with acute gastroenteritis in pediatric patients in Thailand, 2011 to 2017. PLoS One. 2019;14(8):e0220263. https://doi.org/10.13 71/journal.pone.0220263.

9. Russell WC. Adenoviruses: update on structure and function. J Gen Virol. 2009;90(Pt 1):1-20. https://doi.org/10.1099/vir.0.003087-0.

10. Dehghan S, Seto J, Liu EB, Ismail AM, Madupu R, Heim A, et al. A zoonotic adenoviral human pathogen emerged through genomic recombination among human and nonhuman simian hosts. J Virol. 2019;93(18):e00564-19.

11. Tian X, Fan Y, Wang C, Liu Z, Liu W, Xu Y, et al. Seroprevalence of neutralizing antibodies against six human adenovirus types indicates the low level of herd immunity in young children from Guangzhou, China. Virol Sin. 2020;36(3):373-81. https://doi.org/10.1007/s12250-020-00307-1.

12. Adhikary AK, Inada T, Banik U, Numaga J, Okabe N. Identification of subgenus C adenoviruses by fiber-based multiplex PCR. J Clin Microbiol. 2004:42(2):670-3. https://doi.org/10.1128/JCM.42.2.670-673.2004.

13. Lynch JP 3rd, Kajon AE. Adenovirus: epidemiology, global spread of novel serotypes, and advances in treatment and prevention. Semin Respir Crit Care Med. 2016;37(4):586-602. https://doi.org/10.1055/s-003 6-1584923.

14. Lu L, Jia R, Zhong H, Xu M, Su L, Cao L, et al. Molecular characterization and multiple infections of rotavirus, norovirus, sapovirus, astrovirus and adenovirus in outpatients with sporadic gastroenteritis in Shanghai, China, 2010-2011. Arch Virol. 2015;160(5):1229-38. https://doi.org/10.1007/s00705015-2387-1.

15. Afrad MH, Avzun T, Haque J, Haque W, Hossain ME, Rahman AR, et al. Detection of enteric- and non-enteric adenoviruses in gastroenteritis patients, Bangladesh, 2012-2015. J Med Virol. 2018;90(4):677-84. https://doi. org/10.1002/jmv.25008.

16. Ramani S, Kang G. Viruses causing childhood diarrhoea in the developing world. Curr Opin Infect Dis. 2009;22(5):477-82. https://doi.org/10.1097/QCO. ob013e328330662f.

17. de Oliveira Ferreira CE, Raboni SM, Aparecida Pereira L, Nogueira MB, Renaud Vidal LR, Almeida SM. Viral acute gastroenteritis: clinical and epidemiological features of co-infected patients. Braz J Infect Dis. 2012;16(3): 267-72. https://doi.org/10.1016/S1413-8670(12)70322-7.

18. Primo D, Pacheco GT, Timenetsky M, Luchs A. Surveillance and molecular characterization of human adenovirus in patients with acute gastroenteritis in the era of rotavirus vaccine, Brazil, 2012-2017. J Clin Virol. 2018;109:35-40. https://doi.org/10.1016/j.jcv.2018.10.010.

19. Nadan S, Taylor MB, Groome MJ, Cohen C, Madhi SA, Page NA. Epidemiology of human astroviruses among children younger than 5 years: prospective hospital-based sentinel surveillance in South Africa, 2009-2014. J Med Virol. 2019;91(2):225-34. https://doi.org/10.1002/jmv.25308.

20. Bosch A, Pintó RM, Guix S. Human Astroviruses. Clin Microbiol Rev. 2014; 27(4):1048-74. https://doi.org/10.1128/CMR.00013-14.

21. Mitui MT, Bozdayi G, Matsumoto T, Dalgic B, Nishizono A, Ahmed K. Complete genome sequence of an MLB2 Astrovirus from a Turkish child with diarrhea. Genome Announc. 2013;1(4):e00619-3. 
22. Wohlgemuth N, Honce R, Schultz-Cherry S. Astrovirus evolution and emergence. Infect Genet Evol. 2019;69:30-7. https://doi.org/10.1016/j. meegid.2019.01.009.

23. Vu DL, Bosch A, Pinto RM, Guix S. Epidemiology of classic and novel human Astrovirus: gastroenteritis and beyond. Viruses. 2017;9(2):33. https://doi.org/1 0.3390/v9020033.

24. Gelaw A, Pietsch C, Liebert UG. Genetic diversity of human adenovirus and human astrovirus in children with acute gastroenteritis in Northwest Ethiopia. Arch Virol. 2019;164(12):2985-93. https://doi.org/10.1007/s00705-01 9-04421-8.

25. Perot P, Lecuit M, Eloit M. Astrovirus diagnostics. Viruses. 2017;9(1):10. https://doi.org/10.3390/v9010010.

26. Bergallo M, Galliano I, Dapra V, Rassu M, Montanari P, Tovo PA. Molecular detection of human Astrovirus in children with gastroenteritis, Northern Italy. Pediatr Infect Dis J. 2018;37(8):738-42. https://doi.org/10.1097/INF. 0000000000001899.

27. Lu L, Zhong H, Xu M, Su L, Cao L, Dong N, et al. Molecular epidemiology of human calicivirus infections in children with acute diarrhea in Shanghai: a retrospective comparison between inpatients and outpatients treated between 2006 and 2011. Arch Virol. 2014;159(7):1613-21. https://doi.org/1 0.1007/s00705-013-1881-6.

28. Lu L, Zhong H, Xu M, Su L, Cao L, Jia R, et al. Genetic diversity and epidemiology of Genogroup II noroviruses in children with acute sporadic gastroenteritis in Shanghai, China, 2012-2017. BMC Infect Dis. 2019;19(1):736. https://doi.org/10.1186/s12879-019-4360-1

29. Wang X, Wei Z, Guo J, Cai J, Chang H, Ge Y, et al. Norovirus activity and genotypes in sporadic acute diarrhea in children in Shanghai during 2014 2018. Pediatr Infect Dis J. 2019;38(11):1085-9. https://doi.org/10.1097/INF. 0000000000002456.

30. Jin Y, Cheng WX, Yang XM, Jin M, Zhang Q, Xu ZQ, et al. Viral agents associated with acute gastroenteritis in children hospitalized with diarrhea in Lanzhou, China. J Clin Virol. 2009;44(3):238-41. https://doi.org/10.1016/j. jcv.2008.12.010.

31. Noel JS, Lee TW, Kurtz JB, Glass RI, Monroe SS. Typing of human astroviruses from clinical isolates by enzyme immunoassay and nucleotide sequencing. J Clin Microbiol. 1995;33(4):797-801. https://doi.org/10.1128/jcm.33.4.797-801.1 995.

32. Sharif N, Parvez AK, Haque A, Talukder AA, Ushijima H, Dey SK. Molecular and epidemiological trends of human bocavirus and adenovirus in children with acute gastroenteritis in Bangladesh during 2015 to 2019. J Med Virol. 2020;92(12):3194-201. https://doi.org/10.1002/jmv.25812.

33. La Rosa G, Della Libera S, Petricca S, laconelli M, Donia D, Saccucci P, et al. Genetic diversity of human adenovirus in children with acute gastroenteritis, Albania, 2013-2015. Biomed Res Int. 2015;2015:142912.

34. Lu L, Zhong H, Xu M, Su L, Cao L, Dong N, et al. Epidemiology and genotyping of human adenovirus in outpatient children with sporadic diarrhea in Shanghai, China, 2012-2016. Chin J Evid Based Pediatr. 2017; 12(5):352-3

35. Kumthip K, Khamrin P, Ushijima H, Maneekarn N. Molecular epidemiology of classic, MLB and VA astroviruses isolated from <5year-old children with gastroenteritis in Thailand, 2011-2016. Infect Genet Evol. 2018;65:373-9. https://doi.org/10.1016/j.meegid.2018.08.024.

36. Zhirakovskaia E, Tikunov A, Tymentsev A, Sokolov S, Sedelnikova D, Tikunova N. Changing pattern of prevalence and genetic diversity of rotavirus, norovirus, astrovirus, and bocavirus associated with childhood diarrhea in Asian Russia, 2009-2012. Infect Genet Evol. 2019;67:167-82. https://doi.org/1 0.1016/j.meegid.2018.11.006.

37. Siqueira JAM, Oliveira DS, Carvalho TCN, Portal TM, Justino MCA, da Silva LD, et al. Astrovirus infection in hospitalized children: molecular, clinical and epidemiological features. J Clin Virol. 2017;94:79-85. https://doi.org/10.1016/ j.jcv.2017.07.014

38. Zaraket H, Abou-El-Hassan H, Kreidieh K, Soudani N, Ali Z, Hammadi M, et al. Characterization of astrovirus-associated gastroenteritis in hospitalized children under five years of age. Infect Genet Evol. 2017;53:94-9. https://doi. org/10.1016/j.meegid.2017.05.016.

39. Jacobsen S, Hohne M, Marques AM, Beslmuller K, Bock CT, Niendorf S. Cocirculation of classic and novel astrovirus strains in patients with acute gastroenteritis in Germany. J Inf Secur. 2018;76(5):457-64.

40. Moyo SJ, Hanevik K, Blomberg B, Kommedal O, Nordbø SA, Maselle S, et al. Prevalence and molecular characterisation of human adenovirus in diarrhoeic children in Tanzania; a case control study. BMC Infect Dis. 2014 14:666.

41. Ouyang Y, Ma H, Jin M, Wang X, Wang J, Xu L, et al. Etiology and epidemiology of viral diarrhea in children under the age of five hospitalized in Tianjin, China. Arch Virol. 2012;157(5):881-7. https://doi.org/10.1007/ s00705-012-1235-9.

42. Banerjee A, De P, Manna B, Chawla-Sarkar M. Molecular characterization of enteric adenovirus genotypes 40 and 41 identified in children with acute gastroenteritis in Kolkata, India during 2013-2014. J Med Virol. 2017;89(4): 606-14. https://doi.org/10.1002/jmv.24672.

43. Garcia-Basteiro AL, Bosch A, Sicuri E, Bayas JM, Trilla A, Hayes EB. Hospitalizations due to rotavirus gastroenteritis in Catalonia, Spain, 20032008. BMC Res Notes. 2011;4(1):429. https://doi.org/10.1186/1756-0500-4-42 9.

44. Shimizu H, Phan TG, Nishimura S, Okitsu S, Maneekarn N, Ushijima H. An outbreak of adenovirus serotype 41 infection in infants and children with acute gastroenteritis in Maizuru City, Japan. Infect Genet Evol. 2007;7(2):279_ 84. https://doi.org/10.1016/j.meegid.2006.11.005.

45. Li L, Shimizu H, Doan LT, Tung PG, Okitsu S, Nishio O, et al. Characterizations of adenovirus type 41 isolates from children with acute gastroenteritis in Japan, Vietnam, and Korea. J Clin Microbiol. 2004;42(9): 4032-9. https://doi.org/10.1128/JCM.42.9.4032-4039.2004.

46. Fukuda S, Kuwayama M, Takao S, Shimazu Y, Miyazaki K. Molecular epidemiology of subgenus $F$ adenoviruses associated with pediatric gastroenteritis during eight years in Hiroshima prefecture as a limited area. Arch Virol. 2006:151(12):2511-7. https://doi.org/10.1007/s00705-006-0816-x.

47. Dey RS, Ghosh S, Chawla-Sarkar M, Panchalingam S, Nataro JP, Sur D, et al. Circulation of a novel pattern of infections by enteric adenovirus serotype 41 among children below 5 years of age in Kolkata, India. J Clin Microbiol. 2011;49(2):500-5. https://doi.org/10.1128/JCM.01834-10.

48. Lu L, Xu J, Zhong H, Su L, Cao L, Xu M. Surveillance and comparison of human astrovirus epidemiology in hospitalized children and outpatients with acute diarrhea in Shanghai. Chin J Infect Dis. 2016;34(8):463-8.

49. Cordey S, Vu DL, Zanella MC, Turin L, Mamin A, Kaiser L. Novel and classical human astroviruses in stool and cerebrospinal fluid: comprehensive screening in a tertiary care hospital, Switzerland. Emerg Microbes Infect. 2017:6(9):e84. https://doi.org/10.1038/emi.2017.71.

50. Kim JS, Lee WJ, Lee SK, Lee EJ, Hyun J, Kim HS, et al. Molecular epidemiology of human Astrovirus in stool samples from patients with acute gastroenteritis in Korea, 2013-2017. Ann Lab Med. 2019;39(4):367-72. https://doi.org/10.3343/alm.2019.39.4.367.

51. Khamrin P, Thongprachum A, Okitsu S, Hayakawa S, Maneekarn N, Ushijima H. Multiple astrovirus MLB1, MLB2, VA2 clades, and classic human astrovirus in children with acute gastroenteritis in Japan. J Med Virol. 2016;88(2):35660. https://doi.org/10.1002/jmv.24337.

\section{Publisher's Note}

Springer Nature remains neutral with regard to jurisdictional claims in published maps and institutional affiliations.
Ready to submit your research? Choose BMC and benefit from:

- fast, convenient online submission

- thorough peer review by experienced researchers in your field

- rapid publication on acceptance

- support for research data, including large and complex data types

- gold Open Access which fosters wider collaboration and increased citations

- maximum visibility for your research: over $100 \mathrm{M}$ website views per year

At $\mathrm{BMC}$, research is always in progress.

Learn more biomedcentral.com/submissions 